\title{
Numerical Analysis of Electromigration in Thin Film VLSI Interconnections
}

\author{
V. Petrescu*, T. Mouthaan $\dagger$, W. Schoenmaker $\ddagger$, S. Angelescu*, R. Vissarion*, \\ G. Dima*, H. Wallinga ${ }^{\dagger}$, M.D. Profirescu* \\ * EDIL Lahoratory, Department of Electronics and Telecommunications, "Politehnica" Univer- \\ sity of Bucharest, Romania. \\ † MESA Research Institute, University of Twente, The Netherlands. \\ $\ddagger$ ASP-TCAD Division, IMEC vzw. Leuven, Belgium.
}

\section{Summary}

Due to the continuing downscaling of the dimensions in VLSI circuits, electromigration is becoming a serious reliability hazard for the circuits. A software tool based on finite element has been developed to solve the two partial differential equations of the two particle vacancy/imperfection model. The simulation results of complex grains structures of Al thin film show a very good match with the experimental results.

\section{Introduction}

Metallization failure in integrated circuits has been a problem in the semiconductor electronics industry ever since the advent of thin film technology. In VLSI circuits the device dimensions are being scaled down and concurrently the dimensions of the interconnection metallization decrease.

On a chip of hundreds of thousands of components, designers have to guarantee that all the transistors operate within their limits of voltage, current and temperature. Even these devices can fail: very slow degradation of the aluminum metallization can occur when current passes through (electromigration). So that in the design phase the electromigration (defined as mass transport due to the momentum exchange resulting from the collision of conducting electrons and the diffusing rnetal atoms) has to be taken into account, since electromigration has become a serious reliability hazerd for VLSI circuits.

In polycrystalline aluminum films it is necessary to take into account the transport which occurs via circuit paths, mostly grain boundaries. The combination between a high driving force for electromigration (from the high current densities) and a high diffusivity (because of the grain boundaries) makes thin film conductors especially susceptible to electromigration damage.

Microstructural defects are related to those caused by localized change in grain size and those formed at the junction of these grains, the so-called "triple points". As a result of change in the metallic ion concentration, especially along the grain boundaries, voids occur in the areas where atomic transport results in mass depletion. As these voids grow in size, a crack develops that eventually leads to the rupture of the conductor line. On the other hand, whiskers and hillocks are formed in the areas of mass accumulation. This second mode of failure may cause short circuit between adjacent or superimposed conductor lines, as well as breakage through passivating layers, with consequent corrosion problems.

\section{Model equations and numerical method}

A model for electromigration effect has been developed at MESA Research Institute, University of Twente, The Netherlands ([1], [2], [5]). This model determines the early resistance change due to electromigration of alurninum film metallization under electrical stress. Typical curves for early resistance change show a relatively fast (exponential) increase at the start of stressing, a further linear increase in resistance and, when the stress current is switched off, an exponential decay and usually a remanent 
resistance change. The early resistance change measurements ([3],[4]) made on $\mathrm{AC}$ Wheatstone bridge have provided input to establish the proposed model.

The model is based on the movement of vacancies in the aluminum crystal structure. The aluminum has a grain structure with high mobility of vacancies near grain boundaries and a low mobility in bulk material. Vacancies are generated by electrical current flux, leaving immobile damaged sites (imperfections) behind.

The generated vacancies are mobile and imperfections are considered to be immobile and to remain at the position of their formation. The total number of vacancies and imperfections determine the resistance of the material.

The flux $(\vec{J})$ of vacancies $(V)$ under the influence of the applied electric field $(\vec{E})$ is given by:

$$
\vec{J}=-D \cdot \nabla V+\frac{V D}{k T} Z^{*} e \vec{E}
$$

where $Z^{*}$ is the effective valence. The diffusion coefficient $D$ is thermally activated:

$$
D=D_{0} \exp \left(-\frac{E_{a}}{k T}\right)
$$

where $E_{a}$ is the activation energy and $D_{0}$ a pre-exponential factor.

The continuity equations are:

- for vacancies:

$$
\frac{\partial V}{\partial t}=-\nabla \cdot \vec{J}-\frac{V-V_{0}}{\tau_{0}}+G(j)
$$

- for imperfections:

$$
\frac{\partial N_{i}}{\partial t}=-\frac{V-V_{0}}{\tau_{0}}+G(j)
$$

$\begin{array}{llll}\text { where: } & N_{i} & \text { concentration of imperfections } & {\left[\mathrm{cm}^{-3}\right]} \\ V & \text { concentration of vacancies } & {\left[\mathrm{cm}^{-3}\right]} \\ \vec{J} & \text { vacancy flux } & {\left[\mathrm{cm}^{-2} \mathrm{~s}^{-1}\right]} \\ G(j) & \text { current density induced generation term } & {\left[\mathrm{cm}^{-3} \mathrm{~s}^{-1}\right]} \\ \tau_{0} & \text { averaged vacancy lifetime } & {[\mathrm{s}]} \\ \vec{j} & \text { current density } & {\left[\mathrm{Acm}^{-1}\right]} \\ V_{0} & \text { initial concentration of vacancies } & {\left[\mathrm{cm}^{-3}\right]}\end{array}$

The current density induced formation term, $G(j)$, is modeled by the equation:

$$
G(j)=G_{0} \cdot|\vec{j}|=G_{0} \cdot j
$$

where $G_{0}$ is a constant.

The bulk properties within the grains are: the electrical resistivity $\rho_{b}$ and the activation energy $E_{b}$. At the grain boundaries these properties change: $\rho_{g b} \gg \rho_{b}$ and $E_{g b} \ll E_{b}$. Both a vacancy and an imperfection will increase scattering and will give a decrease in electron mobility. The resistivity due to increased scattering is modeled by:

$$
\rho=\rho_{0}\left[1+\xi\left(V-V_{0}+N_{i}-N_{i 0}\right)\right]
$$

where: $\quad N_{i 0}$ initial concentration of imperfections

$$
\begin{array}{lll}
N_{i 0} & \text { initial concentration of imperfections } & {\left[\mathrm{cm}^{-3}\right]} \\
\xi & \text { constant } & {[-]} \\
\rho_{0} & \text { function of } \rho_{g b} \text { and } \rho_{b} & {[\Omega \mathrm{cm}]}
\end{array}
$$

The potential distribution is obtained solving the Poisson equation:

$$
\nabla \cdot \vec{j}=0 \quad \Leftrightarrow \quad \nabla \cdot\left(\frac{1}{\rho} \vec{E}\right)=0
$$

In the joint research between EDIL Laboratory and TCAD team from IMEC, Belgium a new software tool (WVEM program) was developed in order to solve the problem of electromigration by means of a finite element discretisation method of the system of nonlinear partial differential equations (3), (4) and (7). 


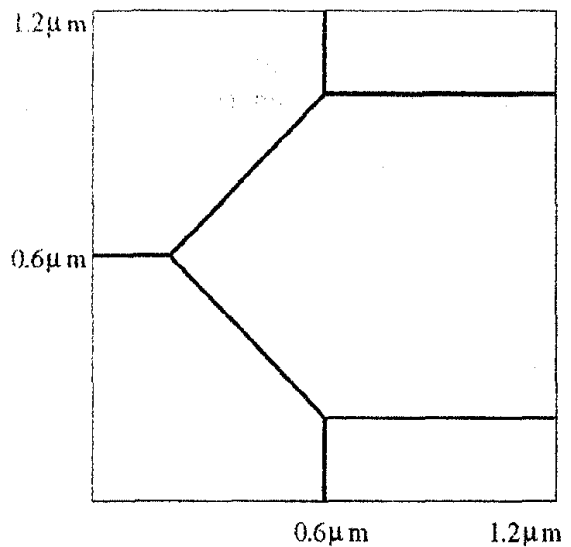

Fig. 1 The simulated structure

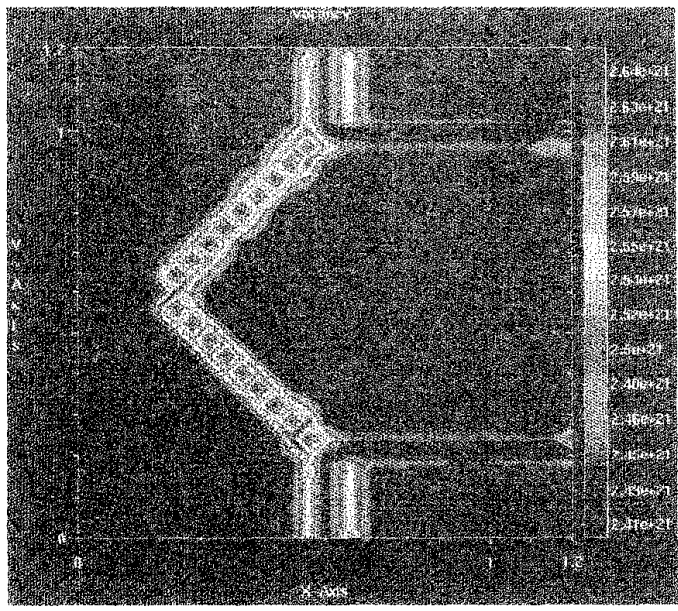

Fig. 3 The vacancy distribution at $t=40 \mathrm{hrs}$

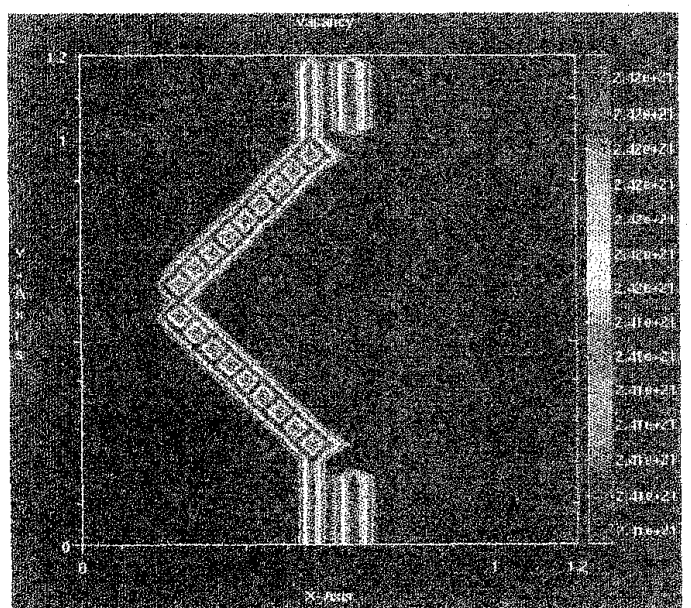

Fig. 5 The vacancy distribution at $t=50 \mathrm{hrs}$

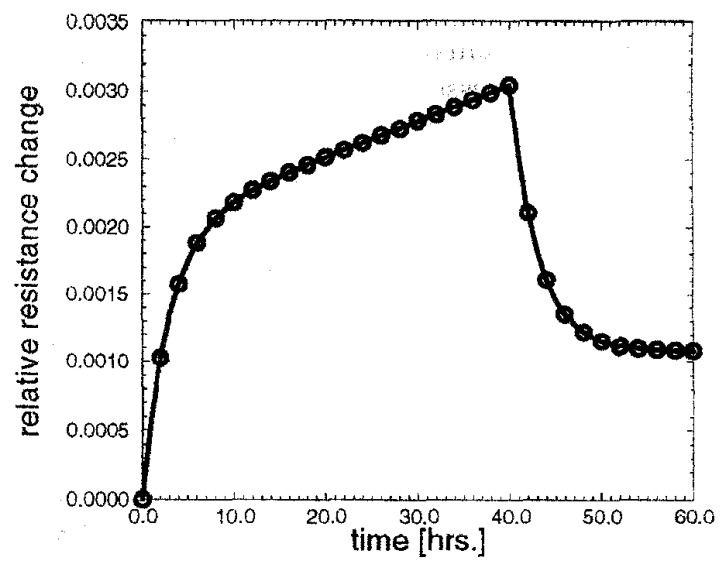

Fig. 2 The relative resistance change

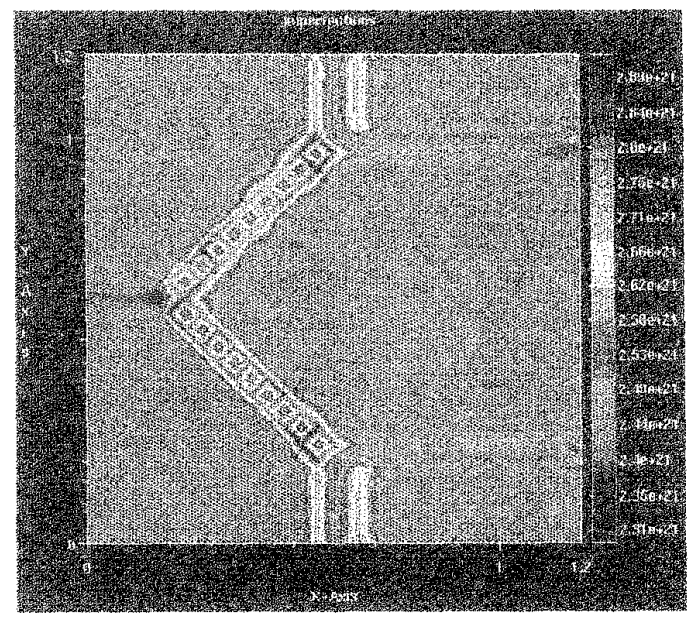

Fig. 4 The imperfection distribution at $t=40 \mathrm{hrs}$

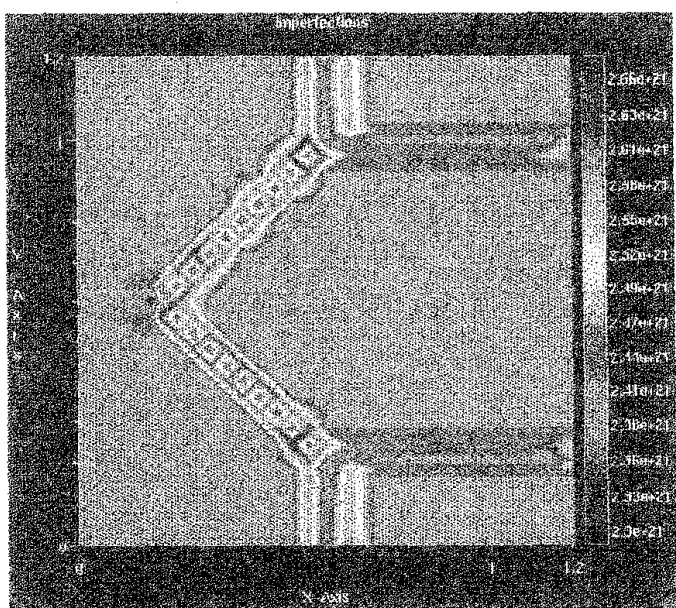

Fig. 6 The imperfection distribution at $t=60 \mathrm{hrs}$ 


\section{Simulation results and discussions}

The simulated domain of a complex triple point grain boundary structure is given in Fig. 1. The structure was stressed with a current density of $400 \mathrm{Acm}^{-1}$ for 40 hours. Then the structure was studied for 20 hours without current stress.

The parameters we used during our simulations are given in Table 1 below:

Table 1: Numerical values for the parameters

\begin{tabular}{|c|c|c|}
\hline Parameter & Value & Units \\
\hline$\rho_{g b}$ & 250 & $\mu \Omega \mathrm{cm}$ \\
$\rho_{b}$ & 2.45 & $\mu \Omega \mathrm{cm}$ \\
$E_{b}$ & 1.4 & $\mathrm{eV}$ \\
$\tau_{0}$ & 3 & hours \\
$G_{0}$ & $2.8 \times 10^{8}$ & $\mathrm{~A}^{-1} \mathrm{~cm}^{-1} \mathrm{~s}^{-1}$ \\
$N_{0}$ & $2.41 \times 10^{21}$ & $\mathrm{~cm}^{-3}$ \\
$V_{0}$ & $2.41 \times 10^{21}$ & $\mathrm{~cm}^{-3}$ \\
$Z^{*}$ & 20 & - \\
$D_{0}$ & 0.1 & $\mathrm{~cm}^{2} \mathrm{~s}^{-1}$ \\
$\theta$ & 25 & ${ }^{\circ} \mathrm{C}$ \\
\hline
\end{tabular}

Fig. 2 gives the relative resistance change of the structure. The relative resistance change after removal of current stress ( $t=40-60$ hours) decays exponentially and saturates at a non-zero level. A permanent resistance change remains as a witness of the permanent damage caused by the 40 hours of current stress.

Fig. 3 and Fig. 4 show the distribution of vacancies and imperfections after 40 hours of current stress. The shape of the two distributions is very similar, but the imperfection distribution is growing continuously. With no stress current at the grain boundary (where the high vacancy mobility implies a fast movement along the boundary) the vacancy distribution is soon becoming flat (see Fig. 5).

Long after removing the applied current ( $t=60$ hours) the concentration of imperfections is high (see Fig. 6) and it can be physically interpreted as a reflection of damage in the grain boundary. structure of the Al thin film.

\section{Conclusions}

The paper presents the numerical implementation of early resistance change of thin film Al interconnections using a model proposed by Niehof and Graaff. A future extension of the model will be an estimation of a link between early resistance change and time-to-failure, in order to be able to use this model for circuits lifetime predictions.

\section{References}

[1] J. Niehof, H.C. de Graaff, J.F. Verweij: Empirical Modeling of Electromigration Early Resistance Changes, Mat. Res. Soc. Symp. Proc. San Francisco, Vol. 309, p. 295-300, 1993.

[2] J. Niehof, H.C. de Graaff, J.F. Verweij: Simulation of Electromigration Early Resistance Changes, Proc. of ESREF'93, p. 135-140, 1993.

[3] J. Niehof, P.A. Flinn, T.J. Maloney: Electromigration Early Resistance Increase Measurements, Proc. of ESREF'92, p. 359-362, 1992.

[4] J. Niehof, P.A. Flinn, T.J. Maloney: Electromigration Early Resistance Increase Measurements, Quality and Reliability International, Vol. 9, No. 4, p. 295-298, 1993.

[5] T. Mouthaan, V. Petrescu, W. Schoenmaker, F. Groot, S. Angelescu, J. Niehof, M.D. Profirescu: Early Resistance Change Modelling in Electromigration, to be published in ESSDERC'95 Proc., 1995 . 